\title{
Diseño e Implementación de Sistemas de Control de Nivel para Cinco Tanques de Bombeo de Hidrocarburos con Sensoramiento tipo Radar en la Estación Shushufindi - EP PETROECUADOR
}

\author{
Hernán Darío Montenegro Cueva, Gustavo David Salazar Chacón
}

\begin{abstract}
Resumen-El presente artículo fue desarrollado para su implementación en fases según el Ciclo de Deming, desarrollando un sistema de control de nivel on-off con histéresis en los tanques de almacenamiento de hidrocarburos en la Estación Cabecera Shushufindi del Poliducto Shushufindi-Quito de EP Petroecuador, teniendo como base de pruebas la Estación Osayacu del mismo poliducto. Además se diseño un sistema totalmente automatizado mediante un control PID como una posible solución al proceso paulatino de modernización de la planta, junto con una propuesta de interfaz humano-máquina (HMI) siguiendo normas ergonómicas de diseño internacional.
\end{abstract}

Palabras Clave-Ciclo PDCA, Control de Nivel, Tanques de Almacenamiento de Hidrocarburos, Automatización de Procesos, Redes Industriales, HART, HMI, Renderización, Recorrido Virtual 3D

\section{INTRODUCCIÓN}

$\mathrm{C}^{\mathrm{L}}$ petróleo, así como sus derivados, son la fuente principal E de energía para muchas actividades humanas de la sociedad actual, tales como la industria, minería y transporte, sin embargo, su explotación, traslado y almacenaje generan problemas ambientales devastadores en caso de existir algún derrame, por ello, tener un sistema de control de nivel robusto es imprescindible para una estación en un poliducto, y es ahí donde reside la importancia del proyecto.

Para conseguir un sistema de control fiable, se plantearon fases de investigación, desarrollo e implementación basados en el Ciclo de Deming (Ciclo PDCA - Plan, Do, Check y Act), consiguiendo de esa manera calidad en la ejecución del proyecto.

Se distinguen tres fases para realizarlo:

- Fase de Investigación y Desarrollo

- Fase de Implementación

- Fase de Desarrollo a Futuro

Hernán Darío Montenegro Cueva, Gustavo David Salazar Chacón; Departamento De Eléctrica y Electrónica, Escuela Politécnica Del Ejercito - ESPE Av. Gral. Rumiñahui s/n Sangolquí - Ecuador; EMails: hd.montenegro@outlook.com, gustavo.salazar@outlook.com

\section{FASE DE INVESTIGACIÓN Y DESARROLLO}

\section{A. Análisis del Estado Actual de la Planta}

Esta etapa permitió tener una visión estructural y técnica del Sistema de Control de Nivel que existía hasta ese momento en la estación, junto con los requerimientos para una modernización de dicho sistema.

1) Revisión del Área Física: Los elementos que formaban parte del Sistema eran (Fig. 1):

- Interruptores de Nivel (Alto y Bajo)

- Bombas de llenado de Hidrocarburo

- Mirillas de Nivel

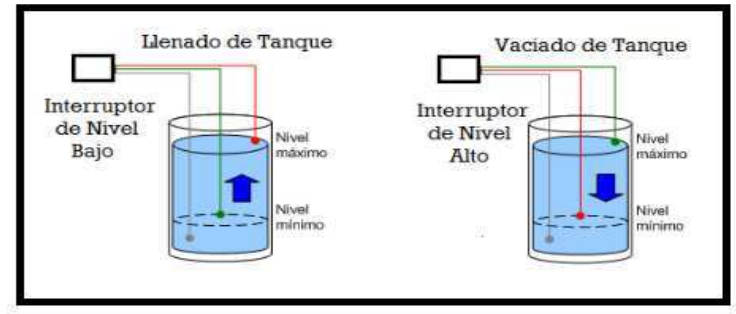

Fig. 1. Principio de funcionamiento sist. de nivel anterior

Básicamente era un sistema sencillo, de fácil instalación y el cual requería poco mantenimiento, pero a la vez tenía falencias en cuanto a tener una medida exacta del nivel, no existía un bus real de sistemas de medición (bus de campo), además de que muchos de esos interruptores se encontraban en mal estado ocasionando medidas erróneas, e incluso no estaban funcionales, lo que obligaba a los operadores a accionar manualmente las bombas según la medida tomada en el mismo tanque a través de las mirillas, por lo que fue necesario una reingeniería del sistema

Apartir de ello, se diseñaron planos eléctricos (Control, Potencia y Entradas Salidas PLC), P\&ID, planos de Tendido de Tubería, Planos Esquemáticos del Sistema de Almacenamiento de Hidrocarburos (Tanques) y Planos 3D de la estación en SolidWorks con el f n de mejorar el desempeo del Sistema de Control de Nivel 
2) Requerimientos Para Modernización: Una vez analizadas las caracteristicas medioambientales (Temperatura, Humedad, Altura, Clima, etc) y visualizar los planos, se identificaron los requerimientos para obtener una modernización del Sistema manteniendo costos no exageradamente elevados, ni cambios drásticos en la infraestructura:

- Al tener distancias que superan los 120 metros entre la fuente de alimentación y el tanque más lejano, se necesita un Sensor que trabaje en el estándar de 4 a 20 [mA] ya que dicho estándar genera poca pérdida de información a largas distancias.

- El control del proceso se realiza en una Plataforma Modicon Quantum, es decir, es necesario el uso de una Red ModBus Plus (MB+) para así mantener coherencia con los datos procesados por el PLC

- Apesar de necesitar una red $\mathrm{MB}+$, es necesario tener comunicación TCP/IP para enviar datos a dispositivos dentro de la Sala de Operaciones como PC, Pantallas Táctiles (Magelis) y Servidores.

- Visualización en tiempo real de los cambios de nivel en cada Tanque.

En base a estos requerimientos el Hardware necesario para implementar el proyecto es el siguiente:

- Radar TDR (Time Domain Ref ectometry) con comunicación en base a Protocolo HART:

Equipo necesario: Radar DR7100 (Fig. 2)

- Pasarela o Gateway de Comunicaciones Industriales Equipo necesario: Prolinx HART-Analog

- Canalización eléctrica subterránea mediante tubos Conduit

- Cableado con protecciones IP66/67 además de ser compatible con señales HART

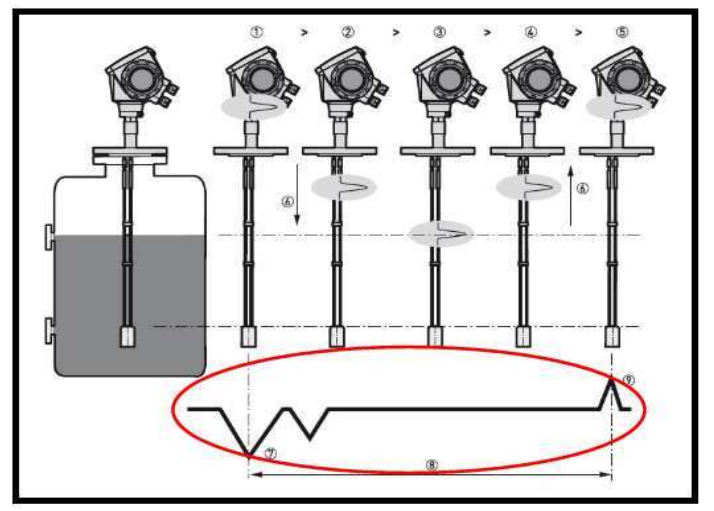

Fig. 2. Medición de nivel con radares DR7100

\section{B. Modelamiento de la Planta en Lazo Abierto}

El Sistema de Almacenamiento de Hidrocarburos de la Estación Shushufindi consta de los Tanques enlistados en la Tabla 1. Además se puede observar una fotografía de la Estación en la Fig. 3:

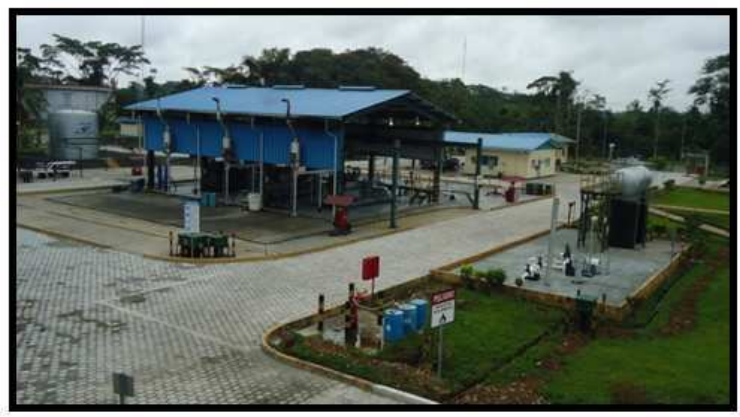

Fig. 3. Estación Shushufindi

TABLA 1

TANQUES - ESTACIÓN SHUSHUFINDI

\begin{tabular}{|c|l|l|c|c|c|}
\hline $\begin{array}{c}\text { Número de } \\
\text { Tanque }\end{array}$ & Geometría & \multicolumn{1}{|c|}{ Producto } & $\begin{array}{c}\text { Altura } \\
(\mathbf{m})\end{array}$ & $\begin{array}{c}\text { Diámetro } \\
(\mathbf{m})\end{array}$ & $\begin{array}{c}\text { Volumen } \\
\left(\mathbf{m}^{3}\right)\end{array}$ \\
\hline 1201 & $\begin{array}{l}\text { Cilindro } \\
\text { Vertical }\end{array}$ & $\begin{array}{l}\text { Almacenamiento } \\
\text { Diesel }\end{array}$ & 5.08 & 3.55 & 61.40 \\
\hline 1202 & $\begin{array}{l}\text { Cilindro } \\
\text { Horizontal }\end{array}$ & $\begin{array}{l}\text { Consumo Diario } \\
\text { Diesel }\end{array}$ & 3.25 & 1.55 & 48.00 \\
\hline 1204 & $\begin{array}{l}\text { Cilindro } \\
\text { Vertical }\end{array}$ & $\begin{array}{l}\text { Alivio Gasolina } \\
\text { Sumidero }\end{array}$ & 3.50 & 2.50 & 50.00 \\
\hline 1205 & $\begin{array}{l}\text { Cilindrico } \\
\text { Horizontal }\end{array}$ & Bullet GLP & 9.25 & 2.10 & 34.70 \\
\hline 1221 & $\begin{array}{l}\text { Cilindro } \\
\text { Vertical }\end{array}$ & $\begin{array}{l}\text { Alivio Gasolina } \\
\text { Base }\end{array}$ & 5.69 & 3.60 & 106.40 \\
\hline
\end{tabular}

El funcionamiento del Sistema de Control de Nivel tiene el siguiente ciclo estructurado (Fig. 4)

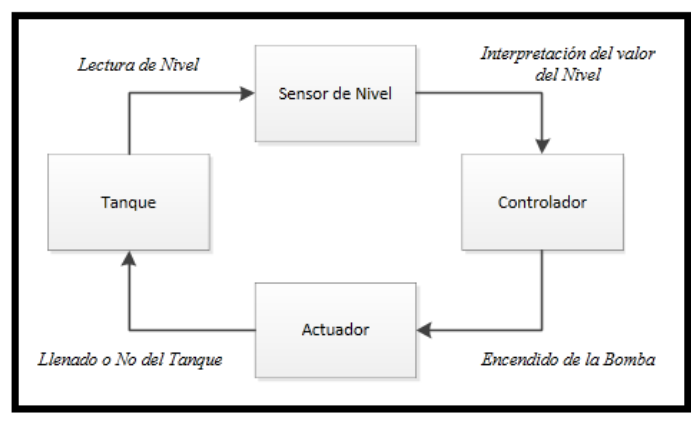

Fig. 4. Ciclo estructurado-control de nivel

1) Identificación del Tipo de Flujo: Una vez entendido el funcionamiento, es necesario identif car el tipo de Flujo que se encuentra en los Tanques de Almacenamiento. Esta aproximación se basa en el Número de Reynolds, si el Flujo es Laminar, debe tener un Número de Reynolds menor a 3000 y Turbulento si es mayor

El hidrocarburo que se encuentra en los Tanques T-1201, T-1202, T-1204 y T-1221 es Gasolina Diesel con las siguientes características:

- Viscosidad cinemática (v): $6.10^{-4}\left[\mathrm{~m}^{2} / \mathrm{s}\right]$

- Caudal que se maneja en el sistema es de $0.0172\left[\mathrm{~m}^{3} / \mathrm{s}\right]$

- Diámetro de la tubería es de una pulgada $(0.0254[\mathrm{~m}])$ 
Diesel

Con estos datos se calcula el Número de Reynolds para

$$
\begin{gathered}
v=\frac{Q}{S}=\frac{0.0172\left[\mathrm{~m}^{3} / \mathrm{s}\right]}{\pi\left(\frac{0.0254[\mathrm{~m}]}{2}\right)^{2}}=33.94[\mathrm{~m} / \mathrm{s}] \\
\operatorname{Re}=\frac{v Q}{v}=\frac{33.94[\mathrm{~m} / \mathrm{s}] 0.0254[\mathrm{~m}]}{6.10^{-4}\left[\mathrm{~m}^{2} / \mathrm{s}\right]}=1436.79 \leq 3000
\end{gathered}
$$

Con lo que se comprueba que el flujo de Gasolina de Diesel es Laminar

2) Identificación de Variables que intervienen en el modelamiento: Las variables que intervienen son (ver Fig. 5):

- Resistencia (R), Variación de la diferencia de nivel necesaria para producir un cambio en el caudal

- Capacitancia del Tanque (C), Variación en la cantidad de líquido acumulado, necesario para producir una variación unitaria en el potencial

- Caudal o Gasto estacionario (Q)

- Mínima desviación en el caudal de entrada con respecto al valor respectivo en estado estacionario (qi)

- Mínima desviación en el caudal de salida con respecto al valor respectivo en estado estacionario (qo)

- Nivel de carga estacionario $(\bar{H})$

- Pequeña desviación del nivel respecto a su valor estacionario (h)

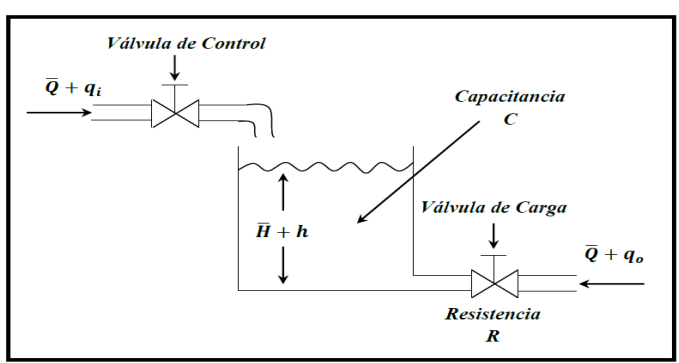

Fig. 5. Modelamiento de tanques - fujo laminar

$$
C d h=(q i-q o) d t
$$

La Resistencia al Flujo se define como una relación entre Caudal y Altura

$$
q o=\frac{h}{R}
$$

Reemplazando la Ecuación 4 en la Ecuación 3 y asignando un valor constante a $\mathrm{R}$ se obtiene:

$$
R C \frac{d h}{d t}+h=R q i
$$

Aplicando la Transformada de Laplace a la Ecuación 5:

$$
\frac{H(s)}{Q(s)}=\frac{R}{R C s+1}
$$

Para determinar el valor de $\mathbf{R}$ - Resistencia, se realizaron pruebas experimentales con el fin de tener la Curva de Nivel
(Fig. 6) en Función del Gasto, pues la pendiente de dicha curva representa a ese valor.

$$
R=\frac{h}{q}=\frac{2 \bar{H}}{\bar{Q}}=P
$$

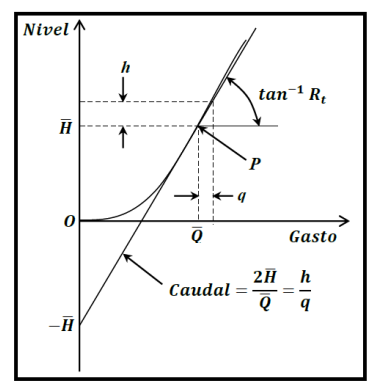

Fig. 6. Curva de nivel en función del gasto

El diagrama de bloques en Lazo Abierto del Sistema de Almacenamiento de Tanques consta de tres partes (Fig. 7):

- Bloque B - Relación entre Voltaje Recibido y Caudal generado por la Bomba Eléctrica de cada Tanque

- Bloque S - Ganancia del Sensor DR-7100

- Función de Transferencia del Tanque

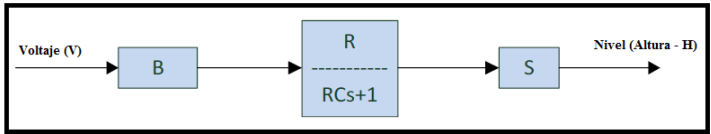

Fig. 7. Diagrama de bloques - lazo abierto

3) Determinación de la Constante $R$ para los Tanques de Almacenamiento: Para cada tanque se registraron datos de llenado y vaciado de hidrocarburo con el fin de generar la curva y obtener la Tangente en el punto de operación de la Bomba.

Para el caso del Tanque Diario T-1202 se realizaron Fig. 8 y Fig. 9

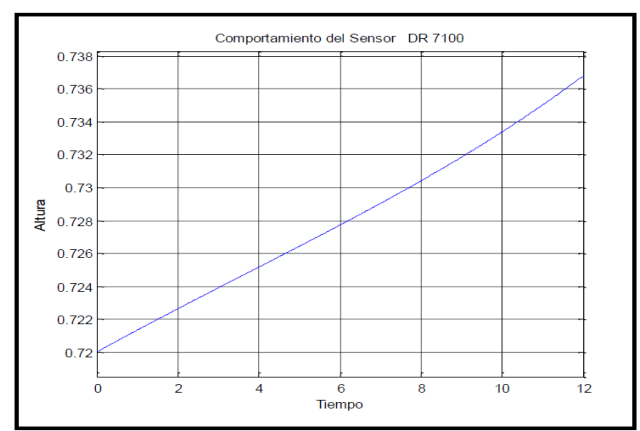

Fig. 8. Comportamiento radar DR 7100 - T-1202 


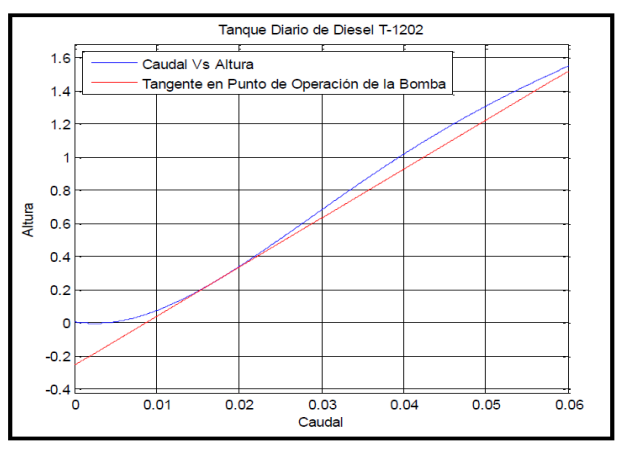

Fig. 9. Curva de nivel en función del gasto - T-1202

De la misma manera, se realizaron las curvas características de cada Tanque del Sistema de Almacenamiento de Hidrocarburos

4) Determinación de la FT en Lazo Abierto de Los Tanques de Almacenamiento de Hidrocarburos: Una vez obtenido los valores necesarios para reemplezar en la Fórmula General del Modelamiento de Tanques (Ecuación 6), se utilizó el Software MatLab y SimuLink para simular el comportamiento de las Plantas en Lazo Abierto (Fig. 10).

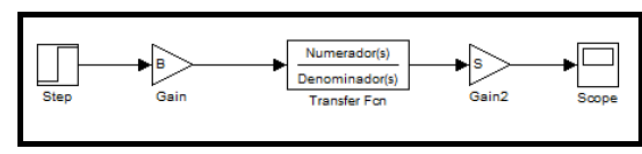

Fig. 10. Simulación Planta Lazo Abierto-SimuLink

Los resultados son los siguientes:

Tanque T-1201 - Almacenamiento de Diesel

Función de Transferencia en Lazo Abierto T-1201:

$$
F T T-1201=\frac{0.0010815}{9025 * s+1}
$$

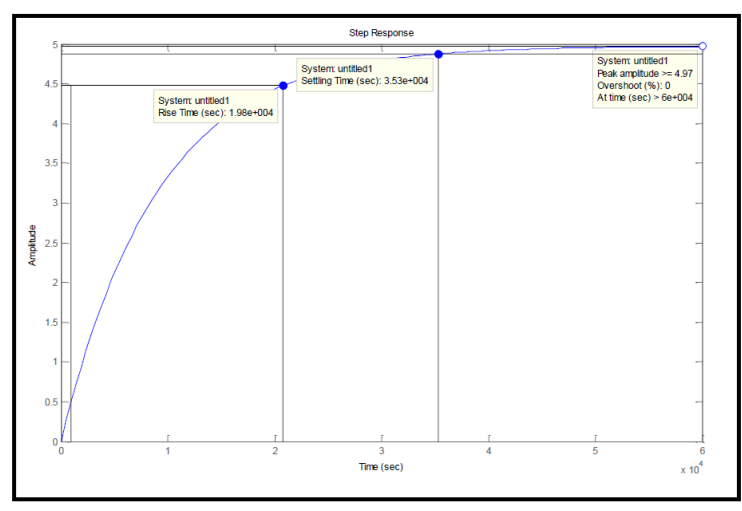

Fig. 11. Respuesta al escalón unitario tanque T-1201
Tanque T-1202 - Diario de Diesel

Función de Transferencia en Lazo Abierto T-1202:

$$
F T T-1202=\frac{0.002459}{913.8 * s+1}
$$

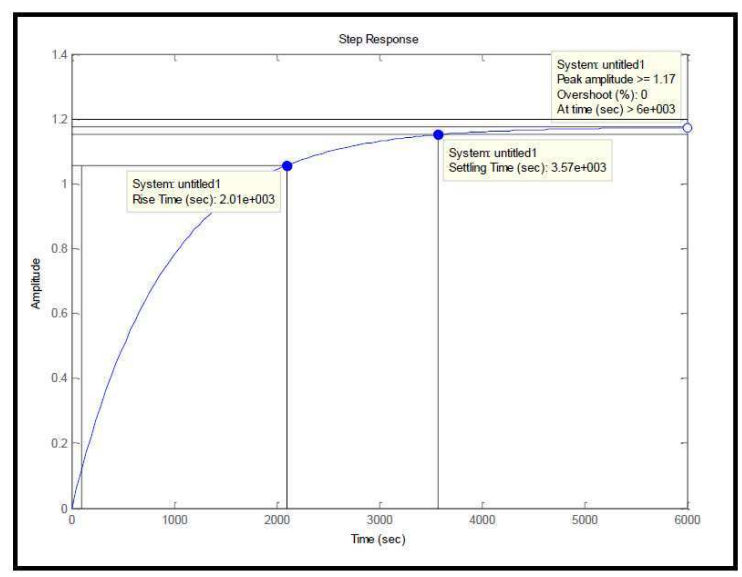

Fig. 12. Respuesta al escalón unitario tanque T-1202

Los demás tanques presentan una respuesta similar, cambiando únicamente tiempos de establecimiento, tiempos de subida y Valor f nal.

Las Funciones de transferencia de los tanques restantes son las siguientes:

Tanque T-1204 - Alivio Gasolina Sumidero

Función de Transferencia en Lazo Abierto T-1204:

$$
F T T-1204=\frac{1.29416}{1.328 * 10^{4} s+1}
$$

Tanque T-1205 - GLP - Bullet

Función de Transferencia en Lazo Abierto T-1205:

$$
F T T-1205=\frac{6.272 * 10^{-5}}{2866 * s+1}
$$

Tanque T-1221 - Alivio Gasolina Base

Función de Transferencia en Lazo Abierto T-1221:

$$
F T T-1221=\frac{0.004350}{551.8 * s+1}
$$

\section{FASE DE IMPLEMENTACIÓN}

Una vez culminada la Fase de Investigación y Desarrollo, en base a los planos diseñados, se implementó el proyecto, en dos etapas:

1) Instalación

2) Puesta en Marcha 


\section{A. Instalación}

La Instalación consiste en ubicar todo lo necesario tanto en Software como en Hardware para llevar a cabo el Proyecto. Entre los puntos más importantes se encuentran (Fig. 13):

- Canalización Eléctrica mediante Tubos Conduit

- Conexión de la Pasarela Prolinx HART-Analog y def nición de Canales HART

- Energización de los Radares DR7100

- Configuraciones de Radares, Pasarela y PLC

- Diseño HMI según normas ergonómicas Internacionales

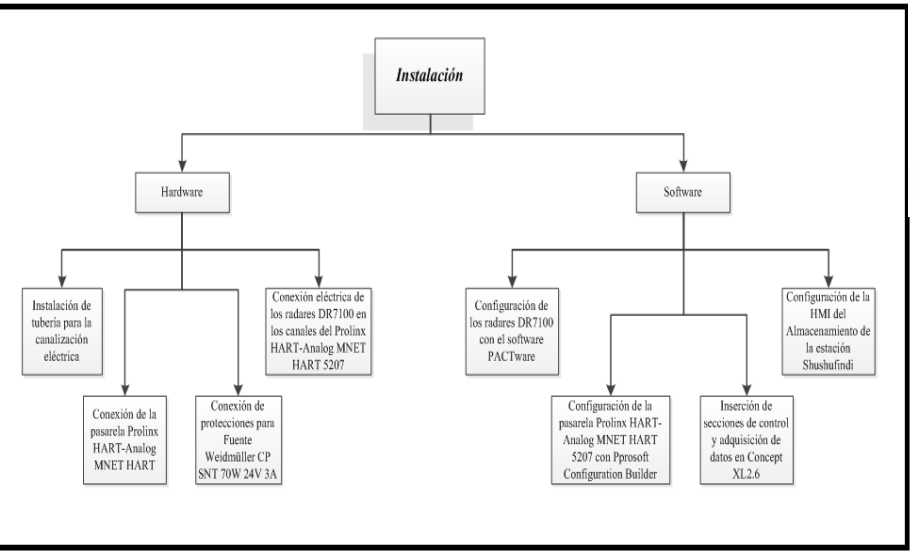

Fig. 13. Mapa conceptual - Instalación

\section{B. Puesta en Marcha}

Una vez realizada la Lógica de Control, HMI, programas de enlace de protocolos de redes Industriales (HARTModbus/TCP), programación de Radares DR 7100 e instalación de equipos siguiendo normas establecidas a nivel internacional como a nivel de EP PETROECUADOR, se realizó la puesta en marcha, para lo cual se cargaron dichos programas en sus respectivos dispositivos.

El siguiente ciclo de proceso (Fig. 14), indica la puesta en marcha del sistema con sus respectivas etapas:

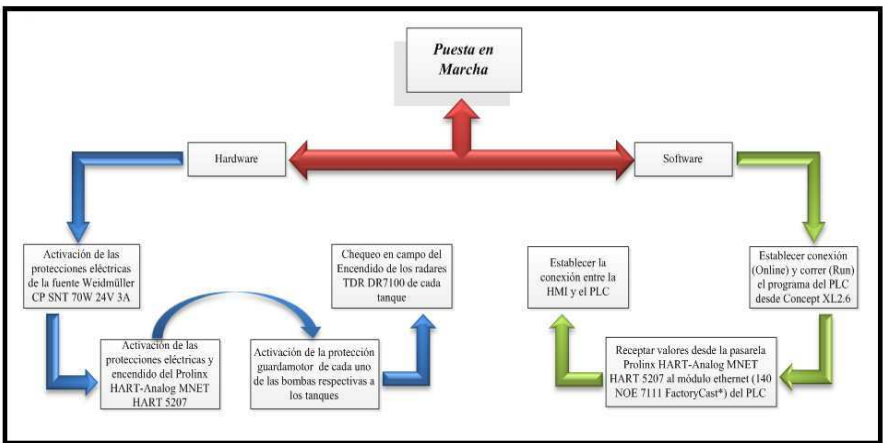

Fig. 14. Ciclo de puesta en marcha del sistema
La HMI diseñada para cumplir con las exigencias de EP Petroecuador bajo normas ergonómicas Internacionales (Guía Gedis) se presenta a continuación en la Fig. 22:

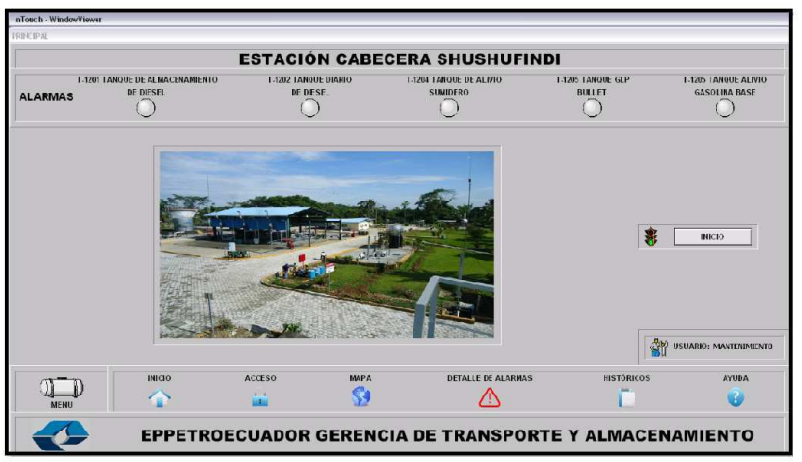

Fig. 15. Pantalla principal HMI

Cuenta con las siguientes pantallas (Fig. 23):

- Control de Acceso (Mantenimiento Eléctrico u Operador)

- Pantalla Principal

- Pantalla Sistema de Almacenamiento de Hidrocarburos (Visualización del estado de la Planta, junto con accesos interactivos mediante esquemas 3D)

- Pantalla de Esquema de Red y Mapa Jerárquico de Distribución de Pantallas (Visualización Interactiva)

- Pantalla de Detalle de Alarmas

- Pantalla de Históricos

- Pantalla de Ayuda

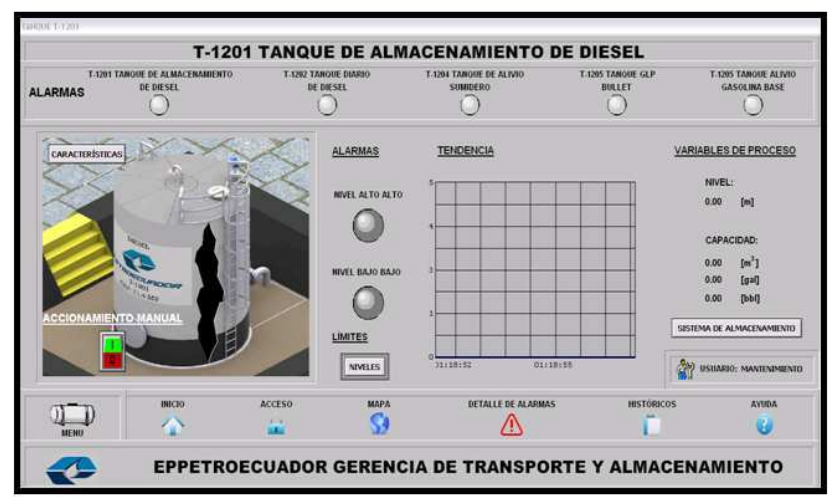

Fig. 16. Pantalla tanque T-1201

Además, en cada una de las pantallas HMI se encuentran las siguientes Barras:

- Barra de Navegación, la cual permite un acceso directo a las pantallas

- Barra de Alarmas Generales, las cuales se activan en caso de existir un estado anómalo en cualquiera de los Tanques 
1) Pruebas: Con el objetivo de verificar conexiones de cable, tendido defectuoso y demás problemas físicos que pudieron darse luego de la ubicación de tubería, equipos y radares, se revisó de manera cuidadosa voltajes, continuidad y protecciones. Además se realizó la prueba final con el Módulo de Prueba en la Estación Shushufindi, dando resultados exitosos. El mapa conceptual que se muestra a continuación (Fig. 17 indica la manera en que se realizaron las pruebas físicas y lógicas del Sistema

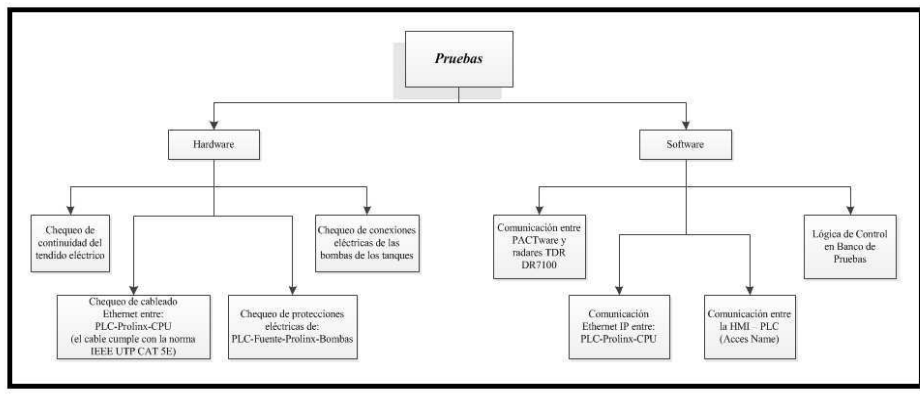

Fig. 17. Pruebas

\section{FASE DE DESARrollo a Futuro}

Con el fin de generar un Control totalmente Automático, se planteó la idea de diseñar un Sistema de Control PID de Nivel en los Tanques de Hidrocarburos de la Estación Shushufindi.

El control automático se basa netamente en la Retroalimentación de la entrada con respecto a la salida con el objeto primordial de generar una acción de control para obtener una respuesta deseada. Actualmente existen novedosas técnicas de Control Moderno como Algoritmos Genéticos, Control Difuso (Fuzzy) o Redes Neuronales, pero el Control PID en los últimos quince años ocupa el $85 \%$ de los bucles de control a nivel industrial, dentro de los cuales alrededor de un $70 \%$ pertenece a un PID clásico - Control PI y un $15 \%$ con variaciones en que se juntan técnicas de Control Adaptativo y Control Inteligente.

\section{A. Diseño Matemático de un Controlador PID para} Tanque Diario T-1202

Para el diseño del controlador se optó por usar un PID en paralelo con el fin de tener independencia en las acciones de control y que cada una de ellas aporte para obtener una respuesta deseada.

Ya determinado el tipo de controlador que se va a diseñar, es necesario el ajuste de los parámetros mediante técnicas de sintonía con el fin de que la respuesta del sistema en lazo cerrado se mantenga bajo criterios de diseño pre-establecidos. Las técnicas empíricas en base a Modelos de ajuste de parámetros están especialmente orientados al mundo industrial, donde existen grandes dificultades para obtener una descripción analítica de los procesos.

Para la sintonización se usaron dos métodos en Lazo Abierto:

- Método de Curva de Reacción Ziegler-Nichols

- Método de Curva de Reacción Cohen-Coon
1) Método de Curva de Reacción Ziegler-Nichols: La planta (Tanque Diario T-1202, Bomba y Radar) puede ser descrita satisfactoriamente mediante el siguiente modelo:

$$
\frac{H(s)}{V(s)}=\frac{(R * B * S) e^{-L S}}{R C s+1}=\frac{K e^{-L S}}{T s+1}
$$

Donde:

- R es la constante que representa la Válvula de Carga del Fluido

- C es la capacitancia del Tanque

- B es la constante que representa el comportamiento de la bomba

- S es la constante que representa el comportamiento del sensor

- L es el tiempo de Retardo o tiempo muerto (Para estudio L es 150 segundos)

Reemplazando dichos valores en la ecuación 13 con los obtenidos en el Modelamiento del Tanque en Lazo Abierto, se obtiene la siguiente respuesta, mostrada en la Fig. 18:

$$
\frac{H(s)}{V(s)}=\frac{0.002459}{913.8 s+1}
$$

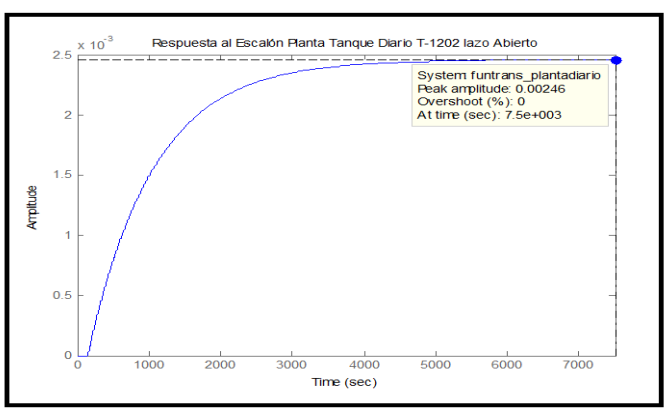

Fig. 18. Respuesta al Escalón Tanque Diario T-1202 Lazo Abierto

En base a la Curva de Ziegler-Nichols y la forma de la Función de Transferencia, se obtienen los siguientes Valores:

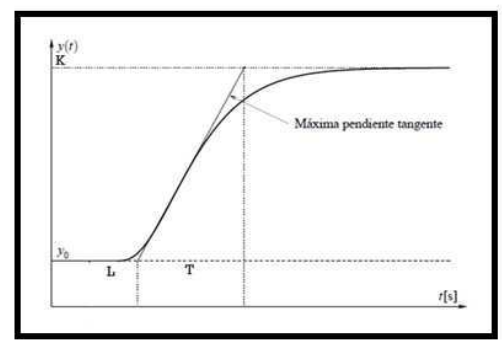

Fig. 19. Curva de Ziegler-Nichols

- $\mathrm{K}=$ Valor Final $=0.002459$

- $\mathrm{L}=$ Tiempo de Retardo $=150$ 
- $\mathrm{T}=$ Cte. de Tiempo $=913.8$

Los parámetros del Controlador PID-Paralelo propuestos por Ziegles-Nichols se observan a continuación (Tabla 2):

TABLA 2

PARÁMETROS DE SINTONIZACIÓN Z-N

\begin{tabular}{|cccc|}
\hline & $\boldsymbol{K}_{p}$ & $\boldsymbol{T}_{r}$ & $\boldsymbol{T}_{d}$ \\
\hline $\mathbf{P}$ & $\frac{\gamma_{0}}{K_{0} \tau_{0}}$ & & \\
\hline PI & $\frac{0.9 \gamma_{0}}{K_{0} \tau_{0}}$ & $3 \tau_{0}$ & \\
\hline PID & $\frac{1.2 \gamma_{0}}{K_{0} \tau_{0}}$ & $2 \tau_{0}$ & $0.5 \tau_{0}$ \\
\hline
\end{tabular}

Por lo que los parámetros sintonizados por Z-N para el controlador PID-Paralelo son:

TABLA 3

PARÁMETROS SINTONIZADOS POR Z-N

\begin{tabular}{|l|c|c|c|}
\cline { 2 - 4 } \multicolumn{1}{c|}{} & $\mathbf{K p}$ & $\mathbf{T i}$ & $\mathbf{T d}$ \\
\hline PID & 2972.9158 & 300 & 75 \\
\hline
\end{tabular}

El Controlador PID paralelo posee la siguiente Forma

$$
\text { ContPID }=K p *\left(1+\frac{1}{T i * s}+T d * s\right)
$$

Reemplazando los parámetros calculados:

$$
\text { ContPID }=\frac{222907.5 s^{2}+2972.91 s+9.09}{s}
$$

Donde la Ecuación 16 representa el Controlador PID Z-N para Tanque Diario T-1202

2) Método de Curva de Reacción Cohen-Coon: Este método de sintonización es similar al de Ziegler-Nichols, la diferencia radica en la Tabla para obtener los parámetros (Tabla 2 y Tabla 4 ).

TABLA 4

\begin{tabular}{|c|c|c|c|}
\hline & $\boldsymbol{K}_{p}$ & $T_{r}$ & $T_{d}$ \\
\hline $\mathbf{P}$ & $\frac{\gamma_{0}}{K_{0} \tau_{0}}\left(1+\frac{\tau_{0}}{3 \gamma_{0}}\right)$ & & \\
\hline PI & $\frac{\gamma_{0}}{K_{0} \tau_{0}}\left(0.9+\frac{\tau_{0}}{12 \gamma_{0}}\right)$ & $\frac{\tau_{0}\left(30 \gamma_{0}+3 \tau_{0}\right)}{9 \gamma_{0}+20 \tau_{0}}$ & \\
\hline & $\frac{\gamma_{0}}{K_{0} \tau_{0}}\left(\frac{4}{3}+\frac{\tau_{0}}{4 \gamma_{0}}\right)$ & $\frac{\tau_{0}\left(32 \gamma_{0}+6 \tau_{0}\right)}{13 \gamma_{0}+8 \tau_{0}}$ & $\frac{4 \tau_{0} \gamma_{0}}{11 \gamma_{0}+2 \tau_{0}}$ \\
\hline
\end{tabular}

SINTONIZACIÓN COHEN-COON

Cohen y Coon desarrollaron una modificación de la tabla de sintonización de Ziegler y Nichols pues determinaron que la Curva de Reacción Z-N es muy sensible a variaciones de $\mathrm{T} / \mathrm{L}$. Cohen y Coon resolvieron este problema de sensibilidad logrando una respuesta más homogénea
Los parámetros sintonizados por C-C para el controlador PID-Paralelo son:

TABLA 5

PARÁMETROS SINTONIZADOS POR C-C

\begin{tabular}{|l|c|c|c|}
\cline { 2 - 4 } \multicolumn{1}{c|}{} & $\mathbf{K p}$ & $\mathbf{T i}$ & $\mathbf{T d}$ \\
\hline PID & 3404.8 & 345.6764 & 52.9647 \\
\hline
\end{tabular}

Reemplazando los parámetros obtenidos en la Ecuación 15:

$$
\text { ContPID }=\frac{180335.895 s^{2}+3404.8 s+9.85}{s}
$$

Donde la Ecuación 17 representa el Controlador PID C-C para Tanque Diario T-1202

\section{B. Simulaciones}

Para comprobar el desempeño de los PID diseñados tanto por el método de sintonización de Ziegler-Nichols y CohenCoon se generó un programa en Matlab y Simulink los cuales simulan el Lazo de Control PID-Paralelo (Ver Fig. 20)

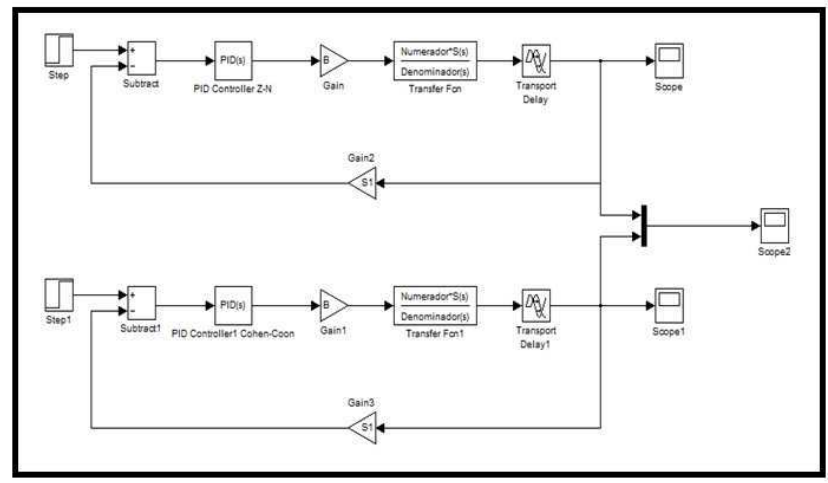

Fig. 20. Programa Simulink lazo de control PID Z-N y C-C

Al analizar el programa en Simulink, se puede ver claramente el Bucle de Control (Realimentado con la señal generada por el Radar DR 7100).

Al igual que en el caso del controlador PID sintonizado mediante el método descrito por Ziegler y Nichols, el controlador C-C (Cohen-Coon) mejora el desempeño general del sistema, disminuyendo el error en estado estacionario y el Tiempo de establecimiento, sin embargo, un mejor control, más estable y menos lento lo realiza el Controlador C-C (ver Fig. 21):

- Tiempo de Establecimiento:

Ts-PIDZN: $1220 \mathrm{seg}$

Ts-PIDCC: $1190 \mathrm{seg}$

$\%$ de MejoraCC: $2.46 \%$ mejor PID-CC frente a PID Z-N

- Tiempo de Subida:

Tr-PIDZN: 264 seg

Tr-PIDCC: 239 seg

\%MejoraCC: $9.47 \%$ mejor PID-CC frente a PID Z-N 


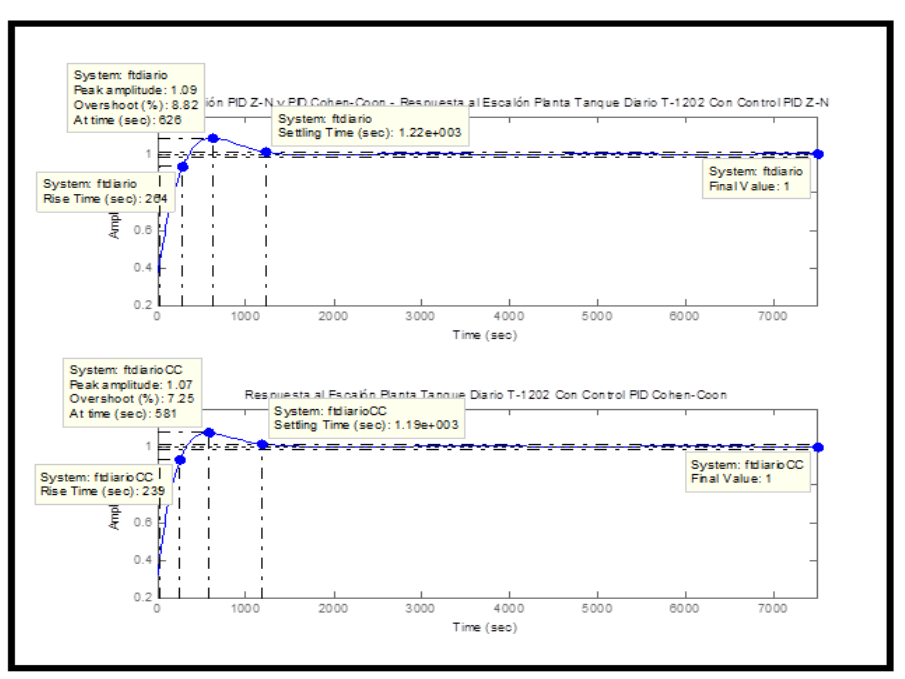

Fig. 21. Respuesta al escalón-Tanque T-1202 PID Z-N y C-C

Por lo visto en los resultados generados, un control PID sintonizado por el método Cohen-Coon genera un $4.5 \%$ de mejora en cuanto al desempeo general frente a un PID sintonizado por el método Ziegler-Nichols.

Cabe indicar, que estas sintonizaciones requieren de una Sintonización Fina para obtener un resultado satisfactorio.

\section{Diseño ESTRUCtURAL Y RECORRIDO 3D}

Con el objetivo de generar una Interface Humano Máquina (HMI) que se capaz de representar todo evento que pueda ocurrir en el proceso de Almacenamiento de Hidrocarburos en los tanques designados, se realizaron planos en tres dimensiones en SolidWorks(3D CAD Design Software) no sólo del Sistema, sino de la estación en su totalidad. Este diseño se puede apreciar en la Fig. 22

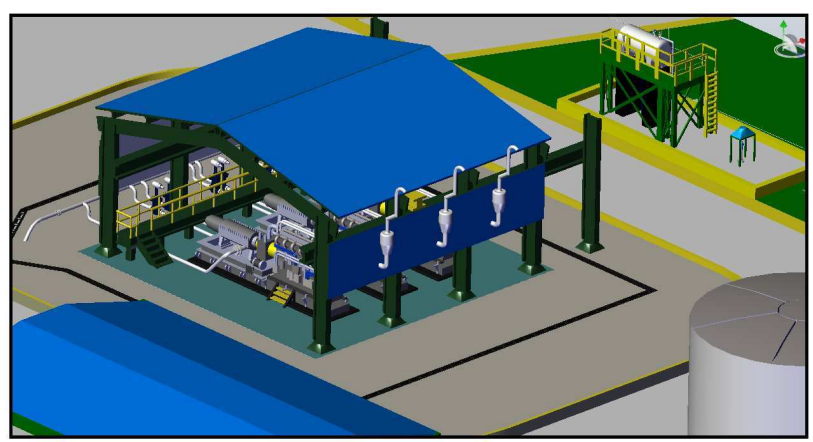

Fig. 22. Plano 3D de la Estación Shushufindi

http://www.solidworks.com/
De esta manera, los operadores podrán tomar mejores decisiones en el proceso ante algún imprevisto debido a que contarán con la mayor cantidad de información posible, tanto visual, como numérica.

Una vez terminados los planos 3D, se diseñó también un Recorrido Virtual 3D (ver Fig. 23 y Fig. 24) tomando como base dichos planos. El recorrido fue realizado en 3DVIA Composer, cuyo principal propósito es ilustrar al personal de la Estación mediante Animaciones Virtuales cualquier eventualidad en el Sistema de Almacenamiento, logrando recrear posibles fallos en ciertas circunstancias extremas (como desbordamientos, falsas alarmas, etc.) y evitar que ocurran en la realidad.

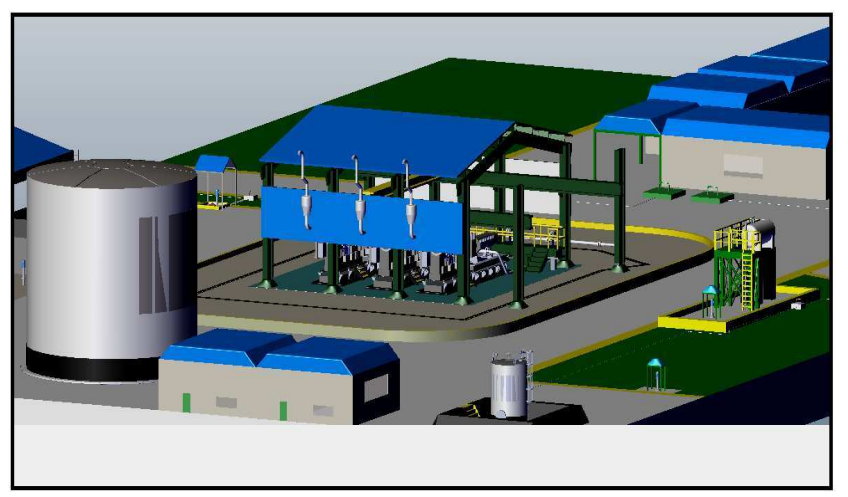

Fig. 23. Recorrido virtual 3D - Estación Shushuf ndi

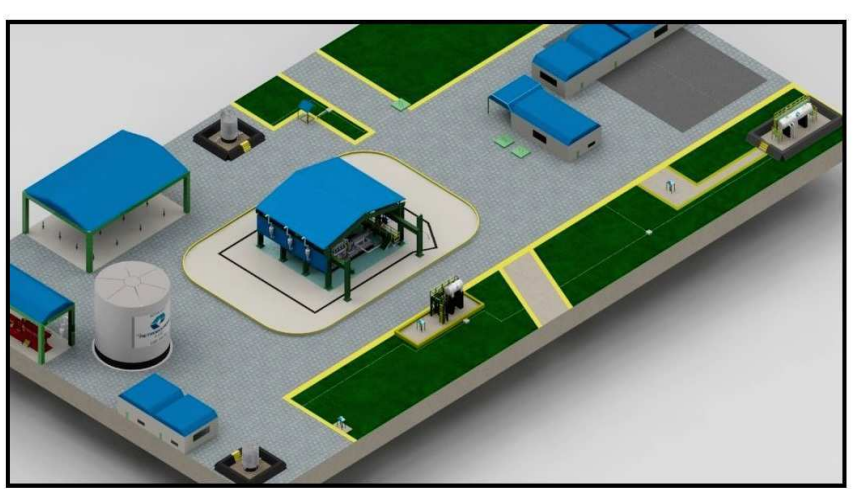

Fig. 24. Recorrido virtual 3D - Estación Shushuf ndi 


\section{CONCLUSIONES}

- Al finalizar el proyecto, se pudo concluir que todo sistema que requiera una mejora en la calidad de procesamiento, reducción de gastos por reparaciones y mayor productividad necesita de una reingeniería, en el caso del Sistema de Control de Nivel era imprescindible una modernización y reestructuración de los dispositivos de campo que median la variable de proceso.

- Con la finalidad de conseguir que la reingeniería del Sistema cumpla con los requerimientos de desempeño, se determinó que deben seguir una metodología sistemática que permita regular el proceso de cambio, para este caso, el método utilizado fue el Ciclo de Deming

- Luego de un estudio de las caracteísticas físicas del entorno, se llegó a la conclusión que el protocolo HART, al utilizar Modulación por desplazamiento de Frecuencia (FSK), reduce el cableado excesivo, genera menos pérdidas de información a largas distancias además de acoplarse ef cientemente a un entorno de Red TCP/IP, es el protocolo de comunicación industrial más adecuado para la implementación del Sistema.

- Los Radares DR 7100 fueron los elegidos para ser los dispositivos de campo al ser muy precisos $( \pm 3$ $[\mathrm{mm}]$ para distancias menores de $10[\mathrm{~m}])$ y tener una resolución de $1[\mathrm{~mm}]$ necesario para tomar datos por fiscalizadores de los Tanques, además de presentar protecciones IP66/67, ser equipo aprueba de explosiones ideal para ambientes con hidrocarburos y principalmente debido a que se comunican al Centro de Control mediante protocolo HART.

- Establecer un control ON - OFF con histéresis para el Sistema de Tanques de Almacenamiento de hidrocarburos genera un mejor uso de los actuadores, ya que al tener un llenado o vaciado paulatino de los tanques, las bombas descansan en periodos de tiempo pre-determinados, alargando su vida útil de operación.

- La ubicación de niveles adicionales de seguridad en la Lógica de Control ON-OFF, generando Bandas de Histéresis secundarias disminuyen la probabilidad a cero de derrame de hidrocarburos.

- Se determinó que al usar un Variador de Velocidad (Driver) es posible tener un sistema totalmente automatizado, liberando al operador de estar siempre activo en la toma de decisiones en el Sistema, evitando de esa manera cualquier posibilidad de derrame al mantener estable el nivel del fuido, lo que conlleva a un costo económico mayor, pero se ve equilibrado por tener siempre la certeza de que dicho fuido se encontrará en el nivel requerido.

- Una HMI correctamente diseñada permite disminuir la presencia de los dos factores más peligrosos para un operador al mando de un sistema: el aburrimiento y la fatiga. Estos dos factores son devastadores, pues ocasionan errores por mal accionamiento de un actuador. Con el objeto de minimizar el efecto nocivo de estos factores siempre presentes, se optó por tener una HMI interactiva con imágenes 3D del proceso, captando de mejor manera la atención de quien opere la HMI.

- Tener restricciones dependiendo el tipo de usuario en la Interfaz Humano Mquina garantiza que no existan cambios inesperados tanto en programación como en Diseño, manteniendo la fdelidad del sistema intacto, permitiendo que sólo personal autorizado y en momentos determinados puedan acceder a la conf guración tanto de la HMI como del Control en sí.

\section{REFERENCIAS}

[1] Trans-Jour.doc, formato de publicacin IEEE Transactions, disponible en: http://www.ieee.org/organizations/pubs/transactions/stylesheets.htm

[2] AMETEK, DREXELBROOK, DR7100 Handbook Guided Radar (TDR) Level Meter for 2-wire Control Systems

[3] OGATA, Katsuhiko, Ingeniera de Control Moderna, 4ta Ed, Prentice Hall, Madrid-Espaa, 2003, ISBN 10: 84-205-3678-4, Págs 135, 137.

[4] RODRÍGUEZ, Aquilino, Sistemas SCADA, 2da Ed., Alfaomega, Mxico D.F.-México, 2006 ISBN: 978-970-15-1305-

[5] BACA, Raúl, Pasado y Futuro del Petróleo en el Ecuador 25 años de exportación del crudo oriente, publicado en 1997 por Unidad de Relaciones Institucionales de Petroecuador.

[6] GÓMEZ GARAY, Vicente, Acciones Básicas de Control, Escuela Superior de Ingenieros de Bilbao, Departamento de Ingeniería de Sistemas y Automática

[7] http://www.inglesa.com.mx /books/DYCTA.pdf, Diseño y Cálculo de Tanques de Almacenamiento 